\title{
On similarity solutions, curved particle motion and artificial gravitation: A case for hydrogen
}

\author{
JM MANALE \\ The Department of Mathematical Sciences \\ University of South Africa \\ Florida, 1709, Johannesburg, Gauteng Province \\ REPUBLIC OF SOUTH AFRICA
}

\begin{abstract}
We demonstrate that suitable initial conditions exist for a particle motion along a curved path, like a circle, without any external forces acting on it. This we achieve by diverting from the popular practice of describing motions of macroscopic bodies through Newtonian mechanics, and instead opt for quantum mechanics. This we do for the hydrogen atom. The validity of the idea is demonstrated by presenting a formula for $\mathrm{G}$, the universal gravitational constant.
\end{abstract}

Keywords - Gravitation; Schrodinger equation; Symmetry Analysis; Modified Symmetries.

Received: October 15, 2020. Revised: February 9, 2021. Accepted: March 3, 2021. Published: March 16, 2021.

\section{Introduction}

I IR Isaac Newton (1643-1727) proposed centuries ago, in what has become known as his third law of motion, that an action force acting on a body has an equal reaction, acting in the opposite direction. We are of the view that this is a special case result, and that it is not generally true.

We demonstrate that when two objects collide, with one having linear momentum and the other angular, then the principle of conservation of linear momentum will not be observed. This we do through quantum mechanics for hydrogen atoms.

We had hoped to solve the equations through Sophus Lie's symmetry group theoretical methods, unfortunately the pure approach does not allow for super-positions. To circumvent this, we introduce what we call modified Lie symmetries, or simply modified symmetries. We use two concepts to evaluate the resulting integrals. The first makes use of intermediate value theorems, and the second is on differentiable topological manifolds.

\section{The Governing Equations of Motion}

The Schrödinger wave equation for the hydrogen atom with electron mass $m_{e}$ and proton mass $m_{p}$ has the form

$$
\begin{aligned}
& -\frac{\hbar^{2}}{2 m_{e}}\left[\frac{\partial^{2}}{\partial x^{2}}+\frac{\partial^{2}}{\partial y^{2}}+\frac{\partial^{2}}{\partial z^{2}}\right] \psi \\
& -\frac{\hbar^{2}}{2 m_{p}}\left[\frac{\partial^{2}}{\partial X^{2}}+\frac{\partial^{2}}{\partial Y^{2}}+\frac{\partial^{2}}{\partial Z^{2}}\right] \psi \\
& +(V-E) \psi=i \hbar \frac{\partial \psi}{\partial t} .
\end{aligned}
$$

Here $\psi=\psi(t, \mathbf{r}, \mathbf{R})$ is the probability density function, $V$ is the potential, $E$ is the energy level, $\mathbf{r}=(x, y, z)$ is the position of the electron, $\mathbf{R}=(X, Y, Z)$ is that of the proton, and $\hbar$ is the reduced Planck constant.
Since the mass of the proton is much larger than that of the proton, then the electron can be interpreted as moving around around a positive core frozen in place in the static potential field $V(R, r)$. This then splits (1) into

$$
-\frac{\hbar^{2}}{2 m_{p}}\left[\frac{\partial^{2}}{\partial X^{2}}+\frac{\partial^{2}}{\partial Y^{2}}+\frac{\partial^{2}}{\partial Z^{2}}\right] \varphi=i \hbar \frac{\partial \varphi}{\partial t}
$$

and

$$
-\frac{\hbar^{2}}{2 m_{e}}\left[\frac{\partial^{2}}{\partial x^{2}}+\frac{\partial^{2}}{\partial y^{2}}+\frac{\partial^{2}}{\partial z^{2}}\right] \phi+(V-E) \phi=i \hbar \frac{\partial \phi}{\partial t},
$$

where $\psi=\varphi(t, \mathbf{R}) \phi(t, \mathbf{r})$. Equation (2) describes the motion of a free particle, implying that the proton is moving freely. Equation (3), on the other hand, is that of a forced particle.

\subsection{Equation (2)}

Equation (2) is easier to analyze as an Airy-LaguerreGaussian equation. This requires transforming the first two terms of the Laplacian through polar coordinates $X=$ $\rho_{p} \cos \theta_{p}$ and $Y=\rho_{p} \sin \theta_{p}$, while leaving the third as is. That is,

$$
-\frac{\hbar^{2}}{2 m_{p}}\left[\frac{\partial^{2}}{\partial \rho_{p}^{2}}+\frac{1}{\rho_{p}} \frac{\partial}{\partial \rho_{p}}+\frac{1}{\rho_{p}^{2}} \frac{\partial^{2}}{\partial \theta_{p}^{2}}+\frac{\partial^{2}}{\partial Z^{2}}\right] \varphi=i \hbar \frac{\partial \varphi}{\partial t},
$$

or

$$
\frac{\partial \varphi}{\partial t}=\frac{i \hbar}{2 m_{p}}\left(\frac{\partial^{2} \varphi}{\partial \rho_{p}^{2}}+\frac{1}{\rho_{p}} \frac{\partial \varphi}{\partial \rho_{p}}+\frac{1}{\rho_{p}^{2}} \frac{\partial^{2} \varphi}{\partial \theta_{p}^{2}}+\frac{\partial^{2} \varphi}{\partial Z^{2}}\right) .
$$

called the Airy-Laguerre-Gaussian equation. Here $\varphi=$ $\varphi\left(t, \rho_{p}, \theta, Z\right)$.

Letting

$$
\varphi=V_{p}\left(t, \rho_{p}\right) \Phi_{p}\left(\theta_{p}\right) P_{p}(t, Z),
$$

separates (16) into three simpler components. This has been executed before, see [1], [2], [3], [4], [5], [6] and [7]. The simplest is the azimuthal equation,

$$
\frac{d^{2} \Phi_{p}}{d \theta_{p}^{2}}+\lambda \Phi_{p}=0
$$


The parameter $m=\sqrt{\lambda}$ is called the azimuthal mode number, also known as the topological charge. Next is the 1D Schrödinger equation,

$$
\frac{\partial P_{p}}{\partial t}-\frac{i \hbar}{2 m_{p}} \frac{\partial^{2} P_{p}}{\partial Z^{2}}=0
$$

and the Laguerre-Scholes equation,

$$
\frac{\partial V_{p}}{\partial t}-\frac{i \hbar}{2 m_{p}}\left(\frac{\partial^{2} V_{p}}{\partial \rho_{p}^{2}}+\frac{1}{\rho_{p}} \frac{\partial V_{p}}{\partial \rho_{p}}-\frac{m_{p}^{2}}{\rho_{p}^{2}} V_{p}\right)=0 .
$$

\subsection{Equation (3)}

Equation (3) transforms through the spherical coordinates $x=\rho_{e} \sin \theta_{p} \cos \sigma_{e}, y=\rho_{e} \sin \theta_{e} \sin \sigma$ and $z=\rho_{e} \cos \theta_{e}$, with $\rho_{e}=\sqrt{x^{2}+y^{2}+z^{2}}$. It transforms into

$$
\begin{aligned}
& \frac{\hbar^{2}}{2 m_{e}}\left[\frac{1}{\rho_{e}^{2}} \frac{\partial}{\partial \rho_{e}}\left[\rho_{e}^{2} \frac{\partial}{\partial \rho_{e}}\right]+\frac{1}{\rho_{e}^{2} \sin \theta} \frac{\partial}{\partial \theta}+\frac{1}{\rho_{e}^{2} \sin ^{2} \theta} \frac{\partial}{\partial \sigma}\right] \phi \\
& +\frac{\hbar^{2}}{2 m_{e}}(V-E) \phi=-i \hbar \frac{\partial \phi}{\partial t} .
\end{aligned}
$$

Letting $\phi=\Theta_{e}(\sigma) R_{e}\left(r_{e}\right) V_{e}\left(\theta_{e}\right)$ separates it into

$$
\begin{gathered}
\frac{d^{2} \Theta_{e}}{d \sigma^{2}}=-m_{l_{e}}^{2} \Theta_{e} \\
\frac{1}{2 m_{e} r_{e}^{2}} \frac{d}{d r_{e}}\left(r_{e}^{2} \frac{d R_{e}}{d r_{e}}\right)-\left(V-2 E-\frac{l_{e}\left(l_{e}+1\right)}{2 m_{e} r_{e}^{2}}\right) R_{e}=0
\end{gathered}
$$

It is assumed that $\phi_{t}=-i E \phi / \hbar$. In the case of a coulombic force, we have

$$
V=-\frac{1}{4 \pi \epsilon_{0}} \frac{q Q}{r_{e}}
$$

so that

$$
\begin{aligned}
& \frac{1}{2 m_{e} r_{e}^{2}} \frac{d}{d r_{e}}\left(r_{e}^{2} \frac{d R_{e}}{d r_{e}}\right) \\
& +\left(\frac{1}{4 \pi \epsilon_{0}} \frac{q Q}{r_{e}}+2 E+\frac{l_{e}\left(l_{e}+1\right)}{2 m_{e} r_{e}^{2}}\right) R_{e}=0,
\end{aligned}
$$

where $\epsilon_{0}$ is the permittivity of free space, $q$ and $Q$ are charges.

The third equation is

$\frac{1}{\sin \theta_{e}} \frac{d}{d \theta_{e}}\left(\sin \theta_{e} \frac{d V_{e}}{d \theta_{e}}\right)-\left(l_{2}\left(l_{2}+1\right)+\frac{m_{l_{e}}^{2}}{\sin ^{2} \theta_{e}}\right) V_{e}=0$.

\section{Solving (2), the Free Particle Wave Equation}

Being able to solve the Schrödinger equation exactly, is the key to particle states, their superpositions and entanglements. These assist in the transition from classical to quantum computing; allowing space for faster computers.

However, there are beliefs that this equation can be solved exactly only for the simplest of systems, such as the particle in a box problem, the Hydrogen atoms and the free particle, and not for complex systems. While we may agree with Toli and Zou [8] that that is the case and that multi-electron systems may probably be unsolvable, we argue here that even the simplest case, the free particle, was not solved correctly.
As a precursor to exact solutions, we begin by first pointing out the mis-steps. In literature, (2) is presented in the form

$$
\frac{\partial u}{\partial \zeta}=\frac{i}{2}\left(\frac{\partial^{2} u}{\partial R^{2}}+\frac{1}{R} \frac{\partial u}{\partial R}+\frac{1}{R^{2}} \frac{\partial^{2} u}{\partial \varphi^{2}}+\frac{\partial^{2} u}{\partial T^{2}}\right)
$$

and called the Airy-Laguerre-Gaussian equation. It models the propagation of energy packets in free space. Here $u=$ $u(\zeta, R, \varphi, T)$ is the complex envelope of the optical field, $R=$ $\sqrt{X^{2}+Y^{2}}=r / r_{0}$ is the dimensionless spatial coordinate, $T=t / t_{0}$ the retarded time, $\zeta=z /\left(k r_{0}^{2}\right)$ is the longitudinal propagation distance, $k=2 \pi N / \lambda_{0}$ is the wave number, with the wavelength $\lambda_{0}$, refractive index $N$ and $\varphi$ the azimuthal angle. The quantities $r_{0}$ and $t_{0}$ are scaling parameters.

Letting

$$
u(\zeta, R, \varphi, T)=V(\zeta, R) \Phi(\varphi) P(\zeta, T),
$$

separates (16) into three simpler components. This has been executed before, see [1], [2], [3], [4], [5], [6] and [7]. The simplest is the azimuthal equation,

$$
\frac{d^{2} \Phi}{d \varphi^{2}}+\lambda \Phi=0
$$

The parameter $m=\sqrt{\lambda}$ is called the azimuthal mode number, also known as the topological charge. Next is the 1D Schrödinger equation,

$$
\frac{\partial P}{\partial \zeta}-\frac{i}{2} \frac{\partial^{2} P}{\partial T^{2}}=0
$$

and the Laguerre-Scholes equation,

$$
\frac{\partial V}{\partial \zeta}-\frac{i}{2}\left(\frac{\partial^{2} V}{\partial R^{2}}+\frac{1}{R} \frac{\partial V}{\partial R}-\frac{m^{2}}{R^{2}} V\right)=0 .
$$

It is thought that the azimuthal equation is solved by letting $\phi=\exp (\lambda \varphi)$. This gives $\phi=A \exp (-i \varphi)+B \exp (i \varphi)$, with $A$ and $B$ as constants. This solution is incorrect.

\subsection{A quadrature solution to the azimuthal equation (18)}

Leonhard Euler(1707-1783) introduced the formula

$$
\Phi=e^{r \varphi},
$$

which he purported to be ideal for solving linear ordinary differential equations with constant coefficients. This formula appears in elementary texts on the subject [9] and also in texts on nonlinear differential equations [10]. It can also be found at the core of some advanced theories, see

1) Euler's method: As a first step in applying (21) to (18), we determine $\phi^{\prime \prime}$ :

$$
\Phi^{\prime \prime}=r^{2} e^{r \varphi} .
$$

Substituting (21) and (22) into (18) gives

$$
\left(r^{2}+\lambda\right) e^{r \varphi}=0 \text {. }
$$

Or simply

$$
r^{2}+\lambda=0 \text {. }
$$

For the case $\lambda=-m^{2}$ equation (24) gives

$$
r^{2}-m^{2}=0 \text {. }
$$


That is,

$$
r^{2}=m^{2},
$$

from which

$$
r= \pm m,
$$

so that

$$
\Phi=e^{ \pm m x}
$$

from (21), which is the solution. The accepted form is

$$
\Phi=A e^{-m \varphi}+B e^{m \varphi} .
$$

where $A$ and $B$ are constants.

For the case $\lambda=-m^{2}$ equation (24) gives

$$
r^{2}+m^{2}=0
$$

That is,

$$
r^{2}=-m^{2}
$$

from which

$$
r= \pm \sqrt{-m^{2}}= \pm i m
$$

so that

$$
\Phi=e^{ \pm i m \varphi} .
$$

The accepted form is

$$
\Phi=C \cos (m \varphi)+D \sin (m \varphi),
$$

where $C$ and $D$ are constants.

2) The quadrature solution: Consider (18) in the form

$$
\Phi^{\prime} \Phi^{\prime \prime}=-\lambda \Phi^{\prime} \Phi
$$

or

$$
\Phi^{\prime} \Phi^{\prime \prime}=-\lambda \Phi \Phi^{\prime}
$$

The chain rule allows it to be expressed in the form

$$
\frac{d}{d \varphi}\left[\frac{\left(\Phi^{\prime}\right)^{2}}{2}\right]=-\lambda \frac{d}{d \varphi}\left[\frac{\Phi^{2}}{2}\right] \text {. }
$$

Integrating:

$$
\int \frac{d}{d \varphi}\left[\frac{\left(\Phi^{\prime}\right)^{2}}{2}\right] d x=-\lambda \int \frac{d}{d \varphi}\left[\frac{\Phi^{2}}{2}\right] d \varphi
$$

gives

$$
\frac{\left(\Phi^{\prime}\right)^{2}}{2}=-\lambda \frac{\Phi^{2}}{2}+E,
$$

where $E$ is the constant of integration. That is,

$$
\Phi^{\prime}=\sqrt{2 E-\lambda \Phi^{2}},
$$

or

$$
\frac{d \Phi}{d \varphi}=\sqrt{2 E-\lambda \Phi^{2}} .
$$

Separating the variables and integrating:

$$
\int \frac{d \Phi}{\sqrt{2 E-\lambda \Phi^{2}}}=\int d \varphi \text {. }
$$

That is,

$$
\int \frac{d \Phi}{\sqrt{\lambda} \sqrt{(2 E / \lambda)-\Phi^{2}}}=\int d \varphi
$$

or

$$
\int \frac{d \Phi}{\sqrt{(2 E / \lambda)-\Phi^{2}}}=\sqrt{\lambda} \int d \varphi .
$$

To evaluate (44), we note that $\sin u=v$ can be expressed in the form $u=\arcsin v$, so that $d u / d v$ can be obtained from

$$
\frac{d}{d v}[\sin u]=\frac{d}{d v}[v]=1
$$

That is,

$$
\cos u \frac{d u}{d v}=1
$$

or

$$
\frac{d u}{d v}=\frac{1}{\cos u}=\frac{1}{\sqrt{1-\sin ^{2} u}}=\frac{1}{\sqrt{1-v^{2}}} .
$$

This means from

$$
\frac{1}{\sqrt{(2 E / \lambda)-\Phi^{2}}}=\left[\frac{1}{\sqrt{2 E / \lambda}}\right] \frac{1}{\sqrt{1-(\Phi / \sqrt{2 E / \lambda})^{2}}}
$$

we have $v=\Phi / \sqrt{2 E / \lambda}$ and $\sin u=v$, then

$$
\frac{1}{\sqrt{(2 E / \lambda)-\Phi^{2}}}=\left[\frac{1}{\sqrt{2 E / \lambda}}\right] \frac{1}{\sqrt{1-v^{2}}} .
$$

That is,

$\int \frac{d \Phi}{\sqrt{(2 E / \lambda)-\Phi^{2}}}=\int\left[\frac{1}{\sqrt{2 E / \lambda}}\right] \frac{\sqrt{2 E / \lambda} d v}{\sqrt{1-v^{2}}}=\int \frac{d v}{\sqrt{1-v^{2}}}$

But

$$
\int \frac{d v}{\sqrt{1-v^{2}}}=\frac{d \sin u}{\sqrt{1-(\sin u)^{2}}}=\int \frac{\cos u d u}{\cos u}=\int d u .
$$

Hence,

$$
\int d u=\sqrt{\lambda} \int d \varphi
$$

That is,

$$
u=\sqrt{\lambda} \varphi+F
$$

where $F$ is an integration constant. That is,

$$
\sin u=\sin (\sqrt{\lambda} \varphi+F) .
$$

That is,

$$
v=\sin (\sqrt{\lambda} \varphi+F)=\Phi / \sqrt{2 E / \lambda},
$$

or

$$
\Phi=\sqrt{2 E} \frac{\sin (\sqrt{\lambda}[\varphi+\phi])}{\sqrt{\lambda}} .
$$

where $\phi=F / \sqrt{\lambda}$. That is,

$$
\Phi=\sqrt{2 E} \frac{e^{i(\sqrt{\lambda}[\varphi+\phi])}-e^{-i(\sqrt{\lambda}[\varphi+\phi])}}{2 i \sqrt{\lambda}} .
$$

The expression in (56) and (34) differ, because the latter does not have $\sqrt{\lambda}=m$ in the denominator. In literature (34) is expressed in the form

$$
\Phi=A_{0} e^{i m_{l} \varphi}+A_{0} e^{-i m_{l} \varphi},
$$


which is then reduced to

$$
\Phi=A_{0} e^{i m_{l} \varphi}
$$

with $m_{l}=0, \pm 1, \pm 2, \pm 3, \cdots$. Equivalently, we can also reduce our (56) the same way to get

$$
\Phi=A_{0} \frac{e^{i m_{l} \varphi}}{i m_{l}} .
$$

But this then creates a problem, for it suggests case $m_{l}=$ $\pm 1, \pm 2, \pm 3, \cdots$. That is, the case $m_{l}=0$ appears to be excluded. To avoid this situation from arising, it is best to use (57), as is, without dropping any term. A comparison of our (57) against (34) appears in Figure 1. The plot of our solution reflects normality, in that it is finite.

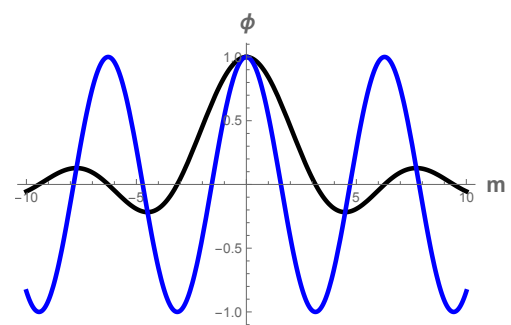

Fig. 1. A plot of our solution in (57), compared to the solution in (34) Ours flattens away from the centre, as expected. The other goes to infinity, which is impractical. The independent variable is constant, and so are the other parameters.

\subsection{Solving the 1D Schrodinger equation (19)}

Our approach to the 1D Schrödinger equation is through Sophus Lie group theoretical methods. We will first demonstrate that the method does lead to an analytical solution, but the solution is not practical. We then modify Lie's method, to deduce a solution that agrees with quantum superposition principles.

1) The pure Lie approach : Lie symmetry is derived from the following definition.

Definition 1: Suppose $x=\left(x^{1}, \cdots, x^{N}\right)$ is a point in $\mathbb{R}^{N}=$ $\mathbb{R} \times \cdots \times N^{t h} \mathbb{R}$, and $\bar{x}=\left(\bar{x}^{1}, \cdots, \bar{x}^{N}\right)$ is a point in $\overline{\mathbb{R}}^{N}=$ $\overline{\mathbb{R}} \times \cdots \times N^{t h} \bar{R}$. The relations

$$
\bar{x}^{i}=f^{i}(x, \epsilon), \quad i=1,2,3, \cdots, N,
$$

where $\epsilon$ is a real parameter, are called a family of one parameter point transformations, if they are invertible.

Equation (19) is much easier to solve in the form

$$
\frac{\partial^{2} \psi}{\partial \tau^{2}}=\frac{\partial \psi}{\partial x}
$$

For it to be so requires setting $i \zeta / 2 \rightarrow x, T \rightarrow \tau$ and $P=\psi$.

To apply Lie's theory to equation (62), we seek to construct the transformations

$$
\begin{aligned}
& \bar{\tau} \approx \tau+\epsilon \xi^{1}(\tau, x, \psi), \\
& \bar{x} \approx x+\epsilon \xi^{2}(\tau, x, \psi), \\
& \bar{\psi} \approx \psi+\epsilon \eta(\tau, x, \psi),
\end{aligned}
$$

with

$$
X=\xi^{1}(\tau, x, \psi) \frac{\partial}{\partial \tau}+\xi^{2}(\tau, x, \psi) \frac{\partial}{\partial x}+\eta(\tau, x, \psi) \frac{\partial}{\partial \psi},
$$

called the symmetry generator. The function $\eta$ can be simplified in advance as $\eta=f(\tau, x) \psi+g(\tau, x)$, so that

$$
X=\xi^{1} \frac{\partial}{\partial \tau}+\xi^{2} \frac{\partial}{\partial x}+(f \psi+g) \frac{\partial}{\partial \psi} .
$$

The second order symmetry generator $X^{[2]}$, derived from it, satisfies the invariance condition

$$
\left.X^{[2]}\left(\psi_{\tau \tau}-\psi_{x}\right)\right|_{\psi_{\tau \tau}=\psi_{x}}=0 .
$$

The analysis of the expanded form of this invariance lead to the following Lie symmetries.

$$
\begin{aligned}
X_{1} & =\frac{x^{2}}{2} \frac{\partial}{\partial x}+\frac{\tau x}{2} \frac{\partial}{\partial \tau}-\left(\frac{\tau^{2}}{8}+\frac{x}{4}\right) \psi \frac{\partial}{\partial \psi} \\
X_{2} & =x \frac{\partial}{\partial x}+\frac{\tau}{2} \frac{\partial}{\partial \tau} \\
X_{3} & =\frac{\partial}{\partial x} \\
X_{4} & =x \frac{\partial}{\partial \tau}-\frac{\tau \psi}{2} \frac{\partial}{\partial \psi} \\
X_{5} & =\frac{\partial}{\partial \tau} \\
X_{6} & =\psi \frac{\partial}{\partial \psi} \\
X_{\infty} & =g \frac{\partial}{\partial \psi} .
\end{aligned}
$$

The symmetry $G_{\infty}$ is called the infinite symmetry, because $g$ is not determined.

2) Solution through $X_{1}$ : Consider the symmetry $X_{1}$. It leads to characteristic equations

$$
\frac{2 d x}{x^{2}}=\frac{2 d \tau}{\tau x}=\frac{d \psi}{-\left(\frac{\tau^{2}}{8}+\frac{x}{4}\right) \psi} .
$$

The characteristic equations yield the invariants

$$
\sigma=\frac{\tau}{x}
$$

and

$$
\phi(\sigma)=\sqrt{x} e^{\frac{\tau^{2}}{4 x}} \psi .
$$

That is,

$$
\psi=\frac{e^{-\frac{\tau^{2}}{4 x}}}{\sqrt{x}} \phi\left(\frac{\tau}{x}\right) .
$$

This leads to

$$
\ddot{\phi}(\sigma)=0,
$$

which has the solution

$$
\phi=D_{1} \sigma+D_{2},
$$

or

$$
\sqrt{x} e^{\frac{\tau^{2}}{4 x}} \psi=D_{1} \frac{\tau}{x}+D_{2} .
$$


That is,

$$
\psi=\left(D_{1} \frac{\tau}{x}+D_{2}\right) \frac{e^{-\frac{\tau^{2}}{4 x}}}{\sqrt{x}},
$$

where $D_{1}$ and $D_{2}$ are constants of integration. That is,

$$
P=\left(D_{1} \frac{T}{i \zeta / 2}+D_{2}\right) \frac{e^{-\frac{T^{2}}{4(i \zeta / 2)}}}{\sqrt{i \zeta / 2}} .
$$

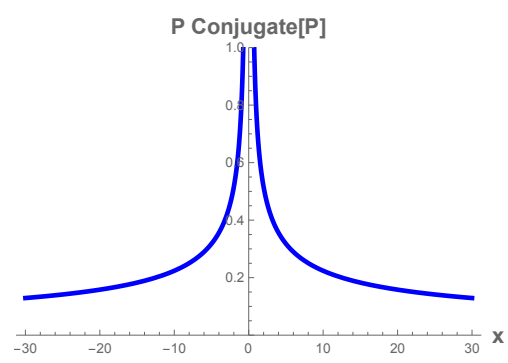

Fig. 2. A plot of the solution in (84), with $D_{1}=D_{2}=1$, and $T=1$.

As indicated earlier, a pure Lie symmetry approach to (19) does not lead to any useful results. We shall now treat it through what we call modified Lie symmetry group theoretical methods.

3) The modified Lie symmetry approach: Our definition of modified Lie symmetry, as contrasted from the pure Lie symmetry Definition 1 , is as follows.

Definition 2: Suppose $G$ is a set of local point transformations from $\mathbb{R}^{N}$ to $\overline{\mathbb{R}}^{N}$, and $\bar{G}$ is a set of local point transformations from $\overline{\mathbb{R}}^{N}$ to $\tilde{\mathbb{R}}^{N}$. Then we call the transformations $\tilde{G}$, from $\mathbb{R}^{N}$ to $\tilde{\mathbb{R}}^{N}$, modified local transformations, if they are invertible.

From this follows the following theorem:

Theorem 1: If $\xi^{i}$ and $\tilde{\xi}^{i}$ are the Lie and modified infinitesimals of the same equation, then

$$
\left.\left(\bar{D}_{j} \bar{\xi}^{i}-D_{j} \xi^{i}\right)\right|_{\omega=0}=0, \quad i, j=1,2,3, \ldots,
$$

where $\omega$ is the parameter for modified symmetries.

Here $\bar{D}_{j}$ and $D_{j}$ are the operators of total differentiation, in their respective spaces. We use it to determine the modified symmetries from Lie's symmetries, one of which is

$$
\begin{aligned}
\tilde{X}_{1}= & \frac{2 e^{\omega^{2} \tau}}{\omega^{4}\left(\omega^{2}-1\right)} \cos \left(\frac{\omega x}{i}\right) \frac{\partial}{\partial \tau} \\
& +\frac{i e^{\omega^{2} \tau}}{\omega^{3}\left(\omega^{2}-1\right)} \sin \left(\frac{\omega x}{i}\right) \frac{\partial}{\partial x} \\
& -\frac{e^{\omega^{2} \tau}}{2} \cos \left(\frac{\omega x}{i}\right) \psi \frac{\partial}{\partial \psi},
\end{aligned}
$$

from which we get the characteristic equation

$$
\begin{aligned}
& \frac{\omega^{4}\left(\omega^{2}-1\right) e^{-\omega^{2} \tau} d \tau}{2 \cos (\omega x / i)}=\frac{i \omega^{3}\left(\omega^{2}-1\right) e^{-\omega^{2} \tau} d x}{\sin (\omega x / i)} \\
& =\frac{2 e^{-\omega^{2} \tau} d u}{\cos (\omega x / i) u} .
\end{aligned}
$$

Consequently, the transforming variables follow from

$$
\frac{\omega^{4}\left(\omega^{2}-1\right) e^{-\omega^{2} \tau} d \tau}{2 \cos (\omega x / i)}=\frac{i \omega^{3}\left(\omega^{2}-1\right) e^{-\omega^{2} \tau} d x}{\sin (\omega x / i)}
$$

and

$$
\frac{\omega^{4}\left(\omega^{2}-1\right) e^{-\omega^{2} \tau} d \tau}{2 \cos (\omega x / i)}=\frac{2 e^{-\omega^{2} \tau} d u}{\cos (\omega x / i) u} .
$$

From (88), we have

$$
\frac{\omega^{2} d \tau}{2}=-\frac{\omega \cos (\omega x / i) d x}{i \sin (\omega x / i)}
$$

That is,

$$
\sigma=\frac{e^{-\omega^{2} \tau / 2}}{\sin (\omega x / i)}
$$

From (89), we have

$$
\frac{2 d u}{u}=\frac{\omega^{4}\left(\omega^{2}-1\right) d \tau}{2},
$$

so that

$$
\phi=u e^{\frac{-\omega^{4}\left(\omega^{2}-1\right) \tau}{4}},
$$

with $\phi=\phi(\sigma)$. Eventually, these transform (19) to

$$
2 \ddot{\phi}\left(e^{\omega^{2} \tau}-\sigma^{2}\right)+\frac{\sigma}{2} \dot{\phi}-\frac{\omega^{2}\left(\omega^{2}-1\right)}{4} \phi=0 .
$$

After a long analysis, we get

$$
\psi=\left(D_{2}+D_{1} \frac{x}{\tau^{3 / 2}}\right) e^{\frac{-x^{2}}{4 \tau}} .
$$

Reverting to the original variables: $x \rightarrow i \zeta / 2, \tau \rightarrow T$ and $\psi \rightarrow P$,

$$
P=\left(D_{2}+D_{1} \frac{i \zeta}{2 T^{3 / 2}}\right) e^{\frac{\zeta^{2}}{16 T}}
$$

Since the particle is in motion, $\mid \zeta>=c T$, with $c$ being its speed, we can have $T=\zeta / c$. This suggests (19) can be written in the form

$$
\frac{\partial P}{\partial T}-i \frac{c^{3}}{2} \frac{\partial^{2} P}{\partial \zeta^{2}}=0
$$

Hence,

$$
P=\left(D_{2}+D_{1} \frac{i c T}{2(\zeta / 2)^{3 / 2}}\right) e^{\frac{c^{3} T^{2}}{16 \zeta}}
$$

A plot similar to the one in Figure 2 is then

The plot also suggests, in the transition from (96) to (98), what the quantum principle states about simultaneity of position, can also be said about time. 


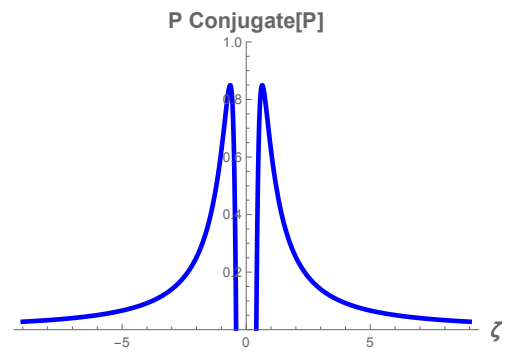

Fig. 3. A plot of the solution in (98), with $D_{1}=D_{2}=1$, and $T=1$. This plot agrees with the quantum superposition principle: The particle is at two positions at the same time.

\subsection{The Airy-Laguerre-Gaussian equation (20)}

A closer look at the Laguerre-Scholes equation reveals a striking resemblance to an equation popular in the mathematics of finance,

$$
V_{\tau_{2}}+\frac{1}{2} A^{2} R^{2} V_{R R}+B R V_{R}-C V=0,
$$

called the Black-Scholes equation. That is,

$$
\frac{\partial V}{\partial \tau_{2}}+R^{2} \frac{\partial^{2} V}{\partial R^{2}}+R \frac{\partial V}{\partial R}-m^{2} V=0
$$

where $\tau_{2}=-i \zeta /\left(2 R^{2}\right) \in R$ in equation (20), $A=\sqrt{2}, b=1$ and $C=m^{2}$. This equation was investigated for solutions, through symmetry methods, by Gazizov and Ibragimov [11] They found the symmetries

$$
\begin{aligned}
X_{1}= & \frac{\partial}{\partial \tau_{2}} \\
X_{2}= & R \frac{\partial}{\partial R} \\
X_{3}= & 2 \tau_{2} \frac{\partial}{\partial \tau_{2}}+\left(\ln R-D \tau_{2}\right) R \frac{\partial}{\partial R} \\
& +2 C \tau_{2} V \frac{\partial}{\partial V} \\
X_{4}= & A^{2} \tau_{2} R \frac{\partial}{\partial R}+\left(\ln R-D \tau_{2}\right) V \frac{\partial}{\partial V} \\
X_{5}= & 2 A^{2} \tau_{2}^{2} \frac{\partial}{\partial \tau_{2}}+2 A^{2} \tau_{2} R \ln R \frac{\partial}{\partial R} \\
+ & \left(\left(\ln R-D \tau_{2}\right)^{2}+2 A^{2} C \tau_{2}^{2}-A^{2} \tau_{2}\right) V \frac{\partial}{\partial V} \\
X_{6}= & V \frac{\partial}{\partial V}, \\
X_{\phi}= & \phi\left(\tau_{2}, R\right) \frac{\partial}{\partial V}
\end{aligned}
$$

where $D=B-A^{2} / 2$ and $\phi\left(\tau_{2}, R\right)$ is an arbitrary solution of (99). For the solution that relates to the Gaussian, they got results from $X_{3}$, and has the form

$$
V=e^{C \tau_{2}} \phi(Z), \quad Z=\frac{\chi}{\sqrt{\tau_{2}}}-D \sqrt{\tau_{2}},
$$

where

$$
\phi(Z)=K_{1} \int_{-\infty}^{Z} e^{\frac{\mu^{2}}{2}} d \mu+K_{2}
$$

where $K_{1}$ and $K_{2}$ are integration constants.
The problem is therefore reduced evaluating the integral

$$
U=\int_{-\infty}^{Z} e^{\frac{\mu^{2}}{2}} d \mu
$$

a form of the Gaussian, with $\phi(Z)=K_{1} U+K_{2}$.

We can express (110) as a differential equation. First we introduce $x$, in the exponent, to ensure we get a partial differential equation. Partial differential equation have many solutions. From

$$
U=\int_{-\infty}^{Z} e^{\frac{t \mu^{2}}{2}} d \mu
$$

we then get

$$
U_{x z}=z^{2} U_{z},
$$

with $U=U(x, z)$. It has only one symmetry

$$
X=\frac{\partial}{\partial x} \text {. }
$$

This we will address through modified symmetries, and they are

$$
\begin{aligned}
\tilde{X}_{1} & =\frac{\sin (\omega) \cos (\omega x)}{\omega} \frac{\partial}{\partial x} \\
& +\ln \left(z^{z}\right)[\sin (\omega) \sin (\omega x) / 2] \frac{\partial}{\partial z} \\
\tilde{X}_{2} & =\frac{\sin (\omega x)}{\omega} \frac{\partial}{\partial x} \\
& -\ln \left(z^{z}\right)[\cos (\omega x) / 2] \frac{\partial}{\partial z} \\
\tilde{X}_{3} & =\ln \left(z^{z}\right) \frac{\partial}{\partial z} \\
\tilde{X}_{4} & =z \frac{\partial}{\partial z}, \\
\text { and } & \\
\tilde{X}_{5} & =u \frac{\partial}{\partial u} .
\end{aligned}
$$

1) The solution through $\tilde{X}_{1}$ :

The characteristic equations that arise from the symmetry $\tilde{X}_{1}$ in (114):

$$
\begin{aligned}
\frac{\omega d x}{\sin (\omega) \cos (\omega x)}= & \frac{d z}{\ln \left(z^{z}\right)[\sin (\omega) \sin (\omega x) / 2]} \\
& =\frac{d v}{0},
\end{aligned}
$$

leads to

$$
\begin{array}{r}
\frac{\omega \sin (\omega x) d x}{2 \cos (\omega x)}=\frac{d z}{\ln \left(z^{z}\right)} \\
=\frac{d v}{0}
\end{array}
$$

The equation

$$
\frac{\omega \sin (\omega x) d x}{2 \cos (\omega x)}=\frac{d z}{\ln \left(z^{z}\right)}
$$

arising from it, integrates into

$$
-\ln [\cos (\omega x)] / 2=\ln [\ln [z]]+d_{2},
$$


or simply

$$
\eta=\sqrt{\cos (\omega x)} \ln [z],
$$

where $\eta$ is an invariant arising out of the integration parameter $d_{1}$. On the other hand, the equation

$$
d v=0,
$$

arising out of the same equation, leads to the invariant

$$
v=\phi(\eta) \text {. }
$$

The invariant $\eta$, leads to

$$
\begin{gathered}
\eta_{z}=\frac{\sqrt{\cos (\omega x)}}{z}, \\
\eta_{x}=\frac{-\omega \sin (\omega x)}{2 \sqrt{\cos (\omega x)}} \ln [z],
\end{gathered}
$$

and

$$
\eta_{x z}=\frac{-\omega \sin (\omega x)}{2 z \sqrt{\cos (\omega x)}}
$$

while $\phi$ gives

$$
\begin{aligned}
v_{z} & =\dot{\phi} \eta_{z} \\
& =\dot{\phi} \frac{\sqrt{\cos (\omega x)}}{z}
\end{aligned}
$$

and

$$
\begin{aligned}
v_{x z}= & \ddot{\phi} \eta_{z} \eta_{x}+\dot{\phi} \eta_{x z} \\
= & -\ddot{\phi} \frac{\sqrt{\cos (\omega x)}}{z} \frac{\omega \sin (\omega x)}{2 \sqrt{\cos (\omega x)}} \ln [z] \\
& -\dot{\phi} \frac{\omega \sin (\omega x)}{2 z \sqrt{\cos (\omega x)}} .
\end{aligned}
$$

The latter simplifies into

$$
v_{x z}=-\ddot{\phi} \frac{\omega \sin (\omega x)}{2 z} \ln [z]-\dot{\phi} \frac{\omega \sin (\omega x)}{2 z \sqrt{\cos (\omega x)}} .
$$

Now substituting (131), and (112) gives

$$
-\ddot{\phi} \frac{\omega \sin (\omega x)}{2 z} \ln [z]-\dot{\phi} \frac{\omega \sin (\omega x)}{2 z \sqrt{\cos (\omega x)}}=z^{2} \dot{\phi} \frac{\sqrt{\cos (\omega x)}}{z} \text {. }
$$

That is,

$$
-\ddot{\phi} \frac{\omega \sin (\omega x)}{2 z} \ln [z]=\dot{\phi} \frac{\omega \sin (\omega x)}{2 z \sqrt{\cos (\omega x)}}+z^{2} \dot{\phi} \frac{\sqrt{\cos (\omega x)}}{z},
$$

or

$$
\frac{\ddot{\phi}}{\dot{\phi}}=\frac{\frac{\omega \sin (\omega x)}{2 z \sqrt{\cos (\omega x)}}+z^{2} \frac{\sqrt{\cos (\omega x)}}{z}}{-\frac{\omega \sin (\omega x)}{2 z} \ln [z]},
$$

or

$$
\ln [\dot{\phi}]=\int \frac{-\frac{\omega \sin (\omega x)}{2 z \sqrt{\cos (\omega x)}}+z^{2} \frac{\sqrt{\cos (\omega x)}}{z}}{-\frac{\omega \sin (\omega x)}{2 z} \ln [z]} d \eta .
$$

The first integrand of this equation integrates easily. That is,

$$
\int \frac{\frac{\omega \sin (\omega x)}{2 z \sqrt{\cos (\omega x)}}}{-\frac{\omega \sin (\omega x)}{2 z} \ln [z]} d \eta=\int \frac{\frac{\omega \sin (\omega x)}{2 z \sqrt{\cos (\omega x)}}}{-\frac{\omega \sin (\omega x)}{2 z} z \ln [z]} \sqrt{\cos (\omega x)} d z
$$

or

$$
\int \frac{\frac{\omega \sin (\omega x)}{2 z \sqrt{\cos (\omega x)}}}{-\frac{\omega \sin (\omega x)}{2 z} \ln [z]} d \eta=\int \frac{-d z}{z \ln [z]}=\ln \left[\frac{1}{\ln [z]}\right]+C_{1},
$$

where $C_{1}$ is an integration constant. The second integrand of (135) requires a different approach to integrate. We note that

$$
\int \frac{z^{2} \frac{\sqrt{\cos (\omega x)}}{z}}{-\frac{\omega \sin (\omega x)}{2 z} \ln [z]} d \eta=\frac{\int \frac{z^{2} \frac{\sqrt{\cos (\omega x)}}{z}}{-\frac{\sin (\omega x)}{2 z} \ln [z]} d \eta}{\omega} .
$$

The expression on the right invokes L'hopital's principle for a zero value of $\omega$ :

$$
\int \frac{z^{2} \frac{\sqrt{\cos (\omega x)}}{z}}{-\frac{\omega \sin (\omega x)}{2 z} \ln [z]} d \eta=\frac{\frac{d}{d \omega} \int \frac{z^{2} \frac{\sqrt{\cos (\omega x)}}{z}}{-\frac{\sin (\omega x)}{2 z} \ln [z]} d \eta}{\frac{d \omega}{d \omega}}
$$

so that

$$
\int \frac{z^{2} \frac{\sqrt{\cos (\omega x)}}{z}}{-\frac{\omega \sin (\omega x)}{2 z} \ln [z]} d \eta=-\frac{\frac{d \eta}{d \omega} \frac{d}{d \eta} \int \frac{z^{2} \frac{\sqrt{\cos (\omega x)}}{z}}{-\frac{\sin (\omega x)}{2 z} \ln [z]} d \eta}{\frac{d \omega}{d \omega}} .
$$

That is,

$\int \frac{z^{2} \frac{\sqrt{\cos (\omega x)}}{z}}{-\frac{\omega \sin (\omega x)}{2 z} \ln [z]} d \eta=-\frac{x \sin (\omega x) \ln [z]}{2 \sqrt{\cos (\omega x)}} \frac{z^{2} \frac{\sqrt{\cos (\omega x)}}{z}}{\frac{\sin (\omega x)}{2 z} \ln [z]}=-t z^{2}$.

Hence,

$$
\ln [\dot{\phi}]=\ln \left[\frac{1}{\ln [z]}\right]+C_{1}-x z^{2}
$$

or

$$
\phi=\int \frac{1}{\ln [z]} \exp \left[C_{1}-x z^{2}\right] d \eta+C_{2} \text {. }
$$

That is,

$$
\begin{aligned}
& \phi=\sqrt{\cos (\omega x)} \ln [\ln [z]] \exp \left[C_{1}-x z^{2}\right] \\
& -\int 2 z^{2} \ln [\ln [z]] \exp \left[C_{1}-x z^{2}\right] d \eta+C_{2} .
\end{aligned}
$$

Hence,

$$
\begin{aligned}
& v=\sqrt{\cos (\omega x)} \ln [\ln [z]] \exp \left[C_{1}-x z^{2}\right] \\
& -\int 2 z^{2} \ln [\ln [z]] \exp \left[C_{1}-x z^{2}\right] d \eta+C_{2} .
\end{aligned}
$$

Applying L'hopital's principles:

$$
\begin{aligned}
& v=\sqrt{\cos (\omega x)} \ln [\ln [z]] \exp \left[C_{1}+x z^{2}\right] \\
& -\omega \frac{d \eta}{d \omega} \frac{d}{d \eta} \int 2 z^{2} \ln [\ln [z]] \exp \left[C_{1}-x z^{2}\right] d \eta \\
& +C_{2} .
\end{aligned}
$$

In the limit $\omega \rightarrow 0$, this reduces to

$$
F=\alpha\left( \pm \ln (\ln [z]) \exp \left(C_{1}+\beta z^{2}\right)+C_{2}\right),
$$

with $\beta$ replacing $x$, and $F / \alpha$ replacing $v$. 


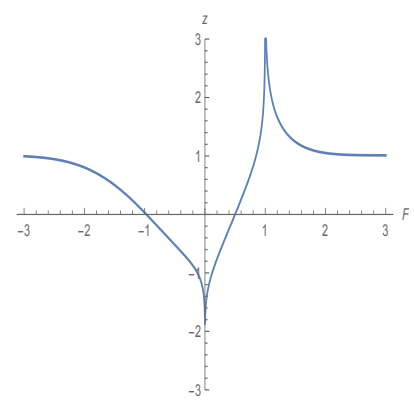

Fig. 4. Plot of the solution in (147), with arbitrary values of $C_{1}$ and $C_{2}$. In this plot, the Gaussian integral is within the interval $0<F<1$. In this here case, the regions $F<0$ and $F>1$ may appear odd. They are unheard of in statistical analysis, where the Gaussian has the biggest role. But they really come into prominence when nonlinear thermal states are investigated.

\section{Solving (3), the Forced Wave Equation}

Letting $\varphi=\varpi(\mathbf{r}) \exp (i E t / \hbar)$ reduces (3) to

$$
-\frac{\hbar^{2}}{2 m_{e}}\left[\frac{\partial^{2}}{\partial x^{2}}+\frac{\partial^{2}}{\partial y^{2}}+\frac{\partial^{2}}{\partial z^{2}}\right] \varpi+(V-2 E) \varpi=0,
$$

a time independent Schrödinger equation.

The substitution $\varpi=R(r) \Theta(\theta) \Phi(\phi)$ reduces it to

$$
\begin{gathered}
\frac{d^{2} \Theta}{d \theta^{2}}=m_{l}^{2} \Theta \\
\frac{1}{2 m r^{2}} \frac{d}{d r}\left(r^{2} \frac{d R}{d r}\right)-\left(V-2 E-\frac{l(l+1)}{2 m r^{2}}\right) R=0 .
\end{gathered}
$$

In the case of a coulombic force, we have

$$
V=-\frac{1}{4 \pi \epsilon_{0}} \frac{q Q}{r}
$$

so that

$$
\frac{1}{2 m r^{2}} \frac{d}{d r}\left(r^{2} \frac{d R}{d r}\right)+\left(\frac{1}{4 \pi \epsilon_{0}} \frac{q Q}{r}+2 E+\frac{l(l+1)}{2 m r^{2}}\right) R=0,
$$

where $\epsilon_{0}$ is the permittivity of free space, $q$ and $Q$ are charges, $r=\|\mathbf{r}\|$ is the relative distance and the position $\mathbf{s}$ of the hydrogen atom is given by its centre of mass:

$$
\mathbf{s}=\frac{m_{e} \mathbf{r}+m_{p} \mathbf{R}}{m_{e}+m_{p}} .
$$

The third equation is

$$
\frac{1}{\sin \theta} \frac{d}{d \theta}\left(\sin \theta \frac{d \Theta}{d \theta}\right)-\left(l(l+1)-\frac{m_{l}^{2}}{\sin ^{2} \theta}\right) \Theta=0 .
$$

\section{A. Solutions through differentiable Manifolds}

\section{Conclusion}

The advantage of having motion without a reaction force has far reaching positive consequences. For example, launching rockets into space will be very cheap, in that far less fuel will be required. It will also realize damages to roads, that automobiles run on, greatly minimized. The reaction force is primary contributor to these damages.

Newtonian mechanics has led to great results and a vast number of technological applications. Unfortunately, a number of confusing concepts have also resulted. Dark matter and energy is one of such concepts. In our view, this results when people have absolute faith in a theory en masse, and get transfixed, probably fueled by what the theorist's background embodies; their numbers then overwhelm logic and common sense. Our suggestion that motion along a curved path is possible without an external force, will probably assist in clearing the confusion.

Gravitation is the most misunderstood concept since Newton's universal gravitational law. A number of theories emerged as efforts to understand it. Ours is yet another addition to this. As a test, in support of ours, we determined the much sought after formula for the universal gravitational constant. In addition, we also proposed frequencies for material elements. The frequencies will assist in harvesting the vast free energy, that is all over the universe, and also in our immediate vicinity.

Militarily, the frequencies can be used in defense systems. To detect and defuse miniaturized weapons, especilly those designed at nano-molecular level.

\section{References}

[1] Zhenkun Wu, Zhiping Wang, Hao Guo, Wei Wang, and Yuzong Gu. Selfaccelerating airy-laguerre-gaussian light bullets in a two-dimensional strongly nonlocal nonlinear medium. Optics Express, 25:30468, 112017.

[2] Yixian Qian and Site Zhang. Quasi-airy beams along tunable propagation trajectories and directions. Optics Express, 24:9489, 52016.

[3] Miao Dong, Xing Yuan Lu, Chengliang Zhao, Yangjian Cai, and Yuanjie Yang. Measuring topological charge of partially coherent elegant laguerre-gaussian beam. Optics Express, 26:033807-1-033806-6, 12 2018.

[4] Lifu Zhang, Kun Liu, Haizhe Zhong, Jinggui Zhang, Ying Li, and Dianyuan Fan. Effect of initial frequency chirp on airy pulse propagation in an optical fiber. Optics Express, 23:2566, 22015.

[5] Dawoon Choi, Kyookeun Lee, Keehoon Hong, Il-Min Lee, KyoungYoum Kim, and Byoungho Lee. Generation of finite power airy beams via initial field modulation. Optics Express, 21:18797, 82013.

[6] Wei-Ping Zhong, Milivoj Beli, and Yiqi Zhang. Three-dimensional localized airy-laguerre-gaussian wave packets in free space. Optics Express, 23:23867, 92015.

[7] A. V. Volyar and T. A. Fadeeva. Laguerre-gaussian beams with complex and real arguments in a uniaxial crystal. Optics and Spectroscopy (English translation of Optika i Spektroskopiya), 101:450-457, 92006.

[8] Ilia Toli and Shengli Zou. Schrdinger equation with coulomb potential admits no exact solutions. Chemical Physics Letters: X, 2, 42019.

[9] Leo J. Grady and Jonathan R. Polimeni. Discrete calculus: Applied analysis on graphs for computational science. Springer London, 2010.

[10] D. W. (Dominic William) Jordan and Peter Smith. Nonlinear ordinary differential equations : an introduction for scientists and engineers. Oxford University Press, 2007.

[11] R K Gazizov and N H Ibragimov. Lie symmetry analysis of differential equations in finance. Nonlinear Dynamics, 17:387-407, 1998.

\section{Creative Commons Attribution License 4.0 (Attribution 4.0 International, CC BY 4.0)}

This article is published under the terms of the Creative Commons Attribution License 4.0 https://creativecommons.org/licenses/by/4.0/deed.en US 